Wiener klinische Wochenschrift The Central European Journal of Medicine

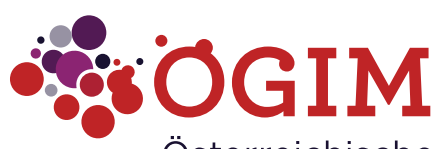

Österreichische Gesellschaft für Innere Medizin

\title{
Korporative Mitglieder der ÖGIM
}

\section{An der Schnittstelle zwischen Industrie, medizinischer Praxis und Forschung}

Die Korporativen Mitglieder der ÖGIM (KM) sind ein Interessensverband, der 2011 in Zusammenarbeit mit der Österreichischen Gesellschaft für Innere Medizin (ÖGIM) gegründet wurde. Die ÖGIM und die KM, derzeit dreizehn Betriebe der pharmazeutische Industrie und Medizintechnikfirmen sowie medizinische Fach- und Zeitschriftenverlage, arbeiten gemeinsam an Projekten der Wissenschafts- und Gesundheitspolitik. Ihr Ziel ist die Zusammenarbeit bei der Förderung von qualitätsvollen und firmenübergreifenden Diskussionen über aktuelle und allgemein relevante medizinisch-wissenschaftliche Themen. So steht etwa die Information über Innovationen in der Forschung und der medizinischen Praxis und über Veränderungen im medizin-technischen Umfeld von Ärzteschaft und Industrie im Mittelpunkt.

Die Leitung der KM wurde seit ihrer Gründung durch ihren Sprecher Roman J. Resch wahrgenommen, der im September 2013 den Stab an Robert Palmetshofer weiter gab. Palmetshofer wird einen nahtlosen Fortgang der bestehenden Aktivitäten garantieren und gleichzeitig eine Schärfung des Profils der KM bewirken.

\section{Schwerpunkt Kommunikation}

Bereits Anfang 2013 beschlossen die KM die Kommunikation der ÖGIM zu unterstützen. In einer mehrteiligen Serie wurden die Mitgliedunternehmen in einem Fachmedium präsentiert. Dabei wurde stets darauf hingewiesen, dass die ÖGIM als Fachgesellschaft nicht nur ein wichtiger Partner der KM ist, sondern auch der größte allgemeine Nenner ihrer Teilgesellschaften darstellt. Mit Beginn des neuen Geschäftsjahres möchte der neue Sprecher der KM diesen Kommunikationsschwerpunkt fortsetzen und gemeinsam mit den Mitgliedsbetrieben um neue Aspekte anreichern. Mit Unterstützung des ÖGIM-Präsidiums wollen sich die KM aber auch verstärkt als Schnittstelle zwischen Industrie, Praxis und Forschung positionieren. Einen ersten Schritt in diese
Richtung setzte ÖGIM-Präsident Dr. Herbert Watzke mit der Einladung an die KM, sich in die Planung der ÖGIMJahrestagung 2014 einzubringen.

\section{Einladung zum Mitmachen}

Alle Interessenten an einer gesundheitspolitischen Diskussion „im Zentrum der Inneren Medizin“ sind herzlich eingeladen, sich den KM anzuschließen.

Kontakt: Geschäftsstelle der Österreichischen Gesellschaft für Innere Medizin, c/o MAW, Gerda Maierhofer, Freyung 6/3, 1010 Wien.

Tel.: 01/536 63-16. E-Mail: oegim@oegim.at

\section{Korporative Mitglieder der ÖGIM Stand März} 2014

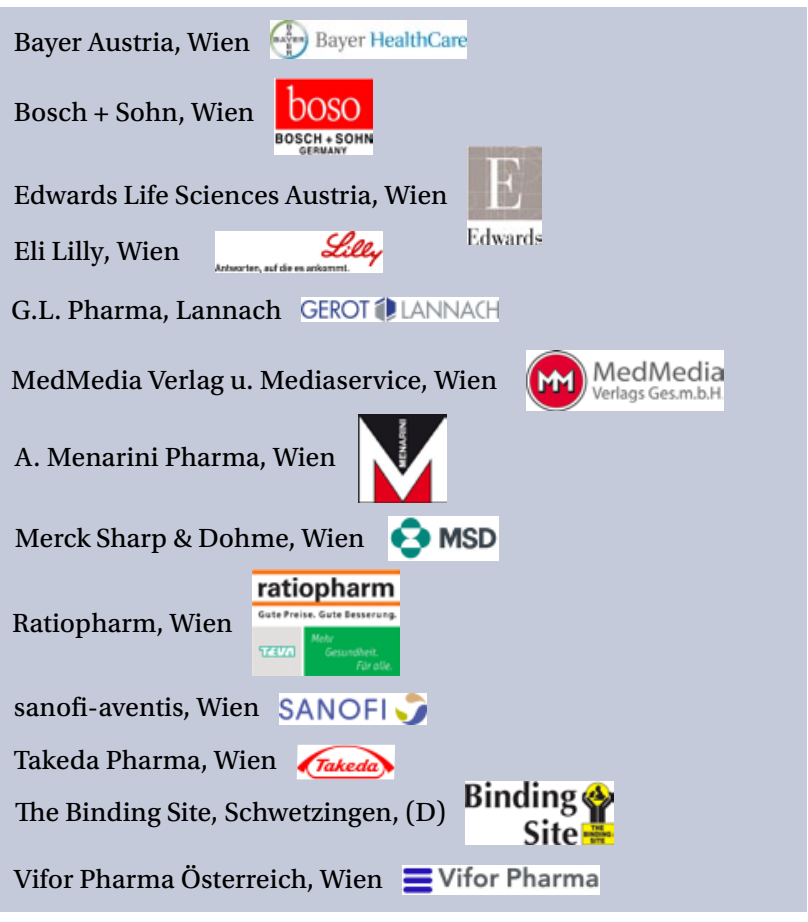

